\title{
Multiplicity of solutions for a $p$-Kirchhoff equation
}

\author{
Jincheng Huang ${ }^{1 *}$, Zhaomin Jiang ${ }^{2}$, Zhiyan $\mathrm{Li}^{1}$ and Jun Wang ${ }^{1}$
}

${ }^{\text {*Correspondence: }}$

slbhuangjc@163.com

${ }^{1}$ Math and Physics Teaching Department, Hohai University,

Changzhou, 213022, China

Full list of author information is

available at the end of the article

\begin{abstract}
In this paper, we consider the following $p$-Kirchhoff equation:

(P) $\quad-\left[M\left(\|u\|^{p}\right)\right]^{p-1} \Delta_{p} u=f(x, u)$ in $\Omega$
\end{abstract}

with Dirichlet boundary conditions, where $\Omega$ is a bounded domain in $\mathbb{R}^{N}$. Under proper assumptions on $M$ and $f$, we obtain three existence theorems of infinitely many solutions for problem $(P)$ by the fountain theorem. Moreover, for a special nonlinearity $f(x, u)=\lambda|u|^{q-2} u+|u|^{r-2} u\left(1<q<p<r<p^{*}\right)$, we prove that problem (P) has at least two nonnegative solutions via the Nehari manifold method and a sequence of solutions with negative energy by the dual fountain theorem.

MSC: 35B38; 35J20; 35J62

Keywords: Kirchhoff equation; fountain theorem; Nehari manifold; fibering map; dual fountain theorem

\section{Introduction}

In this paper, we consider the following $p$-Kirchhoff equation:

$$
-\left[M\left(\|u\|^{p}\right)\right]^{p-1} \Delta_{p} u=f(x, u) \quad \text { in } \Omega, \quad u=0 \quad \text { on } \partial \Omega,
$$

where $M, f$ are continuous functions, $\Omega$ is a bounded domain in $\mathbb{R}^{N}$ with smooth boundary, $\|u\|^{p}=\int_{\Omega}|\nabla u|^{p} d x(1<p<N)$. Let $X$ be the Sobolev space $W_{0}^{1, p}(\Omega)$ endowed with the norm $\|u\|$.

Problem (1.1) began to attract the attention of researchers mainly after the work of Lions [1], where a functional analysis approach was proposed to attack it. Since then, much attention has been paid to the existence of nontrivial solutions, sign-changing solutions, ground state solutions, multiplicity of solutions and concentration of solutions for the following case:

$$
-\left(a+b \int_{\Omega}|\nabla u|^{p} d x\right) \Delta_{p} u=f(x, u) \quad \text { in } \Omega, \quad u=0 \quad \text { on } \partial \Omega .
$$

See $[2-8]$ and the references therein.

For example, $\mathrm{Wu}$ [2] showed that problem (1.2) has a nontrivial solution and a sequence of high energy solutions by using the mountain pass theorem and symmetric

(c) The Author(s) 2017. This article is distributed under the terms of the Creative Commons Attribution 4.0 International License (http://creativecommons.org/licenses/by/4.0/), which permits unrestricted use, distribution, and reproduction in any medium, provided you give appropriate credit to the original author(s) and the source, provide a link to the Creative Commons license, and indicate if changes were made. 
mountain pass theorem. Similar consideration can be found in Nie and Wu [3], where radial potentials were considered. Chen et al. [4] treated equation (1.2) when $f(x, t)=$ $\lambda a(x)|u|^{q-2} u+b(x)|u|^{r-2} u\left(1<q<p=2<r<2^{*}\right)$. Using the Nehari manifold and fibering maps, they established the existence of multiple positive solutions for (1.2).

However, the study of problem (1.1) becomes more difficult since $M$ is a general function. Alves et al. [9] and Corrêa and Figueiredo [10] showed that the problem has a positive solution by the mountain pass theorem, where $M$ is supposed to satisfy the following conditions:

$\left(\mathrm{M}_{1}\right) M(t) \geq m_{0}$ for all $t \geq 0$.

$\left(\mathrm{M}_{2}^{\prime}\right) \hat{M}(t) \geq[M(t)]^{p-1} t$ for all $t \geq 0$, where $\hat{M}(t)=\int_{0}^{t}[M(s)]^{p-1} d s$.

In [11], Liu established the existence of infinite solutions to a Kirchhoff-type equation like (1.1). By the fountain theorem and dual fountain theorem, they investigated the problem with $M$ satisfying $\left(M_{1}\right)$ and

$\left(\mathrm{M}_{3}^{\prime}\right) M(t) \leq m_{1}$ for all $t>0$.

Very recently, Figueiredo and Nascimento [12] and Santos Jr. [13] considered solutions of (1.1) by the minimization argument and the minimax method, respectively, where $p=2$ and $M$ satisfies $\left(\mathrm{M}_{1}\right)$ and

$\left(\mathrm{M}_{4}^{\prime}\right)$ the function $t \mapsto M(t)$ is increasing, and the function $t \mapsto \frac{M(t)}{t}$ is decreasing.

Note that $M(t)=a+b t$ does not satisfy $\left(\mathrm{M}_{2}^{\prime}\right)$ for $p=2$ and $\left(\mathrm{M}_{3}^{\prime}\right)$. Moreover, $M(t)=a+b t^{k}$ does not satisfy $\left(\mathrm{M}_{2}^{\prime}\right),\left(\mathrm{M}_{3}^{\prime}\right)$ for all $k>0$ and $\left(\mathrm{M}_{4}^{\prime}\right)$ for all $k>1$.

Motivated mainly by $[4,5,14]$, we shall establish conditions on $M$ and $f$ under which problem (1.1) possesses infinitely many solutions in the present paper.

Instead of $\left(\mathrm{M}_{2}^{\prime}\right)-\left(\mathrm{M}_{4}^{\prime}\right)$, we make the following assumptions on $M$ :

$\left(\mathrm{M}_{2}\right)$ There exists $\sigma>0$ such that

$$
\hat{M}(t) \geq \sigma[M(t)]^{p-1} t
$$

holds for all $t \geq 0$, where $\hat{M}(t)=\int_{0}^{t}[M(s)]^{p-1} d s$.

$\left(\mathrm{M}_{3}\right)$ There exist $\mu>0, \sigma>0$ and $s>p^{-1}$ such that for all $t \geq 0$

$$
\hat{M}(t) \geq \sigma[M(t)]^{p-1} t+\mu t^{s}
$$

We also suppose that $f$ satisfies the following conditions:

$\left(\mathrm{f}_{1}\right)$ There are constants $1<p<q<p^{*}=\frac{N p}{N-p}$ and $C>0$ such that

$$
|f(x, t)| \leq C\left(1+|t|^{q-1}\right)
$$

for all $x \in \Omega, t \in \mathbb{R}$.

$\left(\mathrm{f}_{2}\right) f(x, t)=o\left(|t|^{p-1}\right)$ as $t \rightarrow 0$ uniformly for any $x \in \Omega$.

$\left(\mathrm{f}_{3}\right) f(x,-t)=-f(x, t)$ for all $x \in \Omega, t \in \mathbb{R}$.

$\left(\mathrm{f}_{4}\right)$ There exists $\frac{p}{\sigma}<\alpha<p^{*}$ such that $0<\alpha F(x, t) \leq t f(x, t)$ for all $x \in \Omega, t \in \mathbb{R}$, where $F(x, t)=\int_{0}^{t} f(x, s) d s$. 
(f $\left.\mathrm{f}_{5}\right)$ There exist $\max \left\{\frac{p}{\sigma}, p\right\}<\alpha<p^{*}$ and $r>0$ such that

$$
\inf _{x \in \Omega,|u|=r} F(x, u)>0
$$

and

$$
0<\alpha F(x, t) \leq t f(x, t)
$$

for all $x \in \Omega$ and $|t| \geq r$.

$\left(\mathrm{f}_{6}\right) 0<\frac{p}{\sigma} F(x, t) \leq t f(x, t)$ holds for all $x \in \Omega, t \in \mathbb{R}$.

(f) $\frac{F(x, t)}{t^{p / \sigma}} \rightarrow+\infty$ as $|t| \rightarrow \infty$ uniformly in $x \in \Omega$.

The associated energy functional to equation (1.1) is

$$
J(u)=\frac{1}{p} \hat{M}\left(\|u\|^{p}\right)-\int_{\Omega} F(x, u) d x .
$$

For any $\phi \in C_{0}^{\infty}(\Omega)$, we have

$$
\left\langle J^{\prime}(u), \phi\right\rangle=\left[M\left(\|u\|^{p}\right)\right]^{p-1} \int_{\Omega}|\nabla u|^{p-2} \nabla u \cdot \nabla \phi d x-\int_{\Omega} f(x, u) \phi d x .
$$

We have the following results by the fountain theorem.

Theorem 1.1 Assume $\left(\mathrm{f}_{1}\right)-\left(\mathrm{f}_{4}\right)$ and $\left(\mathrm{M}_{1}\right)-\left(\mathrm{M}_{2}\right)$. Then problem (1.1) has a sequence $\left\{u_{n}\right\}$ of solutions in $X$ with $J\left(u_{n}\right) \rightarrow \infty$ as $n \rightarrow \infty$.

Theorem 1.2 Assume $\left(\mathrm{f}_{1}\right)-\left(\mathrm{f}_{3}\right),\left(\mathrm{f}_{5}\right)$ and $\left(\mathrm{M}_{1}\right)-\left(\mathrm{M}_{2}\right)$. Then problem $(1.1)$ has a sequence $\left\{u_{n}\right\}$ of solutions in $X$ with $J\left(u_{n}\right) \rightarrow \infty$ as $n \rightarrow \infty$.

Theorem 1.3 Assume $\left(\mathrm{f}_{1}\right)-\left(\mathrm{f}_{3}\right),\left(\mathrm{f}_{6}\right)-\left(\mathrm{f}_{7}\right)$ and $\left(\mathrm{M}_{1}\right),\left(\mathrm{M}_{3}\right)$. Then problem $(1.1)$ has a sequence $\left\{u_{n}\right\}$ of solutions in $X$ with $J\left(u_{n}\right) \rightarrow \infty$ as $n \rightarrow \infty$.

Furthermore, we also consider a special nonlinearity $f(x, u)=\lambda|u|^{q-2} u+|u|^{r-2} u(1<q<$ $\left.p<r<p^{*}\right)$. In this case, the associated energy functional is $J_{\lambda}$ defined by

$$
J_{\lambda}(u)=\frac{1}{p} \hat{M}\left(\|u\|^{p}\right)-\frac{1}{q} \int_{\Omega} \lambda|u|^{q} d x-\frac{1}{r} \int_{\Omega}|u|^{r} d x
$$

where $\hat{M}(s)=\int_{0}^{s}[M(t)]^{p-1} d t$.

Note that this nonlinearity does not satisfy conditions $\left(f_{2}\right),\left(f_{4}\right)-\left(f_{7}\right)$. For this case, we will prove that problem (1.1) has at least two nonnegative solutions by extracting a minimizing sequence from the Nehari manifold, and we will obtain a sequence of weak solutions with negative energy by the dual fountain theorem.

Theorem 1.4 Let $f(x, u)=\lambda|u|^{q-2} u+|u|^{r-2} u$, where $1<q<\min \left\{p, \frac{p}{\sigma}\right\} \leq \max \left\{p, \frac{p}{\sigma}\right\}<r<p^{*}$. Suppose that $M$ satisfies $\left(\mathrm{M}_{1}\right),\left(\mathrm{M}_{2}\right)$ and 
$\left(\mathrm{M}_{4}\right) M$ is differentiable for all $t \geq 0$ and there exist some $d>1$ such that

$$
(r-p) M(t)>d p(p-1) M^{\prime}(t) t \geq 0 .
$$

Then there exists $\lambda_{0}>0$ such that problem (1.1) has at least two nonnegative solutions for all $0<\lambda<\lambda_{0}$.

Theorem 1.5 Let $f(x, u)=\lambda|u|^{q-2} u+|u|^{r-2} u$, where $1<q<\min \left\{p, \frac{p}{\sigma}\right\} \leq \max \left\{p, \frac{p}{\sigma}\right\}<r<p^{*}$. Suppose that $M$ satisfies $\left(\mathrm{M}_{1}\right)$ and $\left(\mathrm{M}_{2}\right)$. Then problem (1.1) has a sequence of solutions $u_{k}$ such that $J_{\lambda}\left(u_{k}\right)<0$ and $J_{\lambda}\left(u_{k}\right) \rightarrow 0$ as $k \rightarrow \infty$.

Remark 1.1 Set $M(t)=a+b t^{k}(a, b, k>0)$. Then we can easily deduce that

(i) $M$ satisfies $\left(\mathrm{M}_{2}\right)$ for all $p>1$ and $0<\sigma \leq \frac{1}{(p-1) k+1}$;

(ii) $M$ satisfies $\left(\mathrm{M}_{3}\right)$ for one of the following cases:

(1) $s=1, p \geq 2,1-\sigma-\sigma(p-1) k \geq 0$, and $0<s \mu \leq(1-\sigma) a^{p-1}$;

(2) $s=k+1, p \geq 2,0<\sigma<1$, and $0<s \mu \leq((1-\sigma) b-\sigma(p-1) b k) a^{p-2}$;

(iii) $M$ satisfies $\left(\mathrm{M}_{4}\right)$ for $r-p>d p k$.

Remark 1.2 Let $M(t)=a+b \ln (1+t)(a, b>0, t \geq 0)$. By direct calculation, one has

$$
\begin{aligned}
\hat{M}(t) & =\int_{0}^{t}(M(t))^{p-1} d t \\
& =t(M(t))^{p-1}-\int_{0}^{t} b(p-1)(M(t))^{p-2} d t+\int_{0}^{t} \frac{b(p-1) M(t)^{p-2}}{1+t} d t \\
& \geq t(M(t))^{p-1}-b(p-1) t M(t)^{p-2} \\
& \geq t(M(t))^{p-1}\left(1-\frac{b(p-1)}{a}\right) .
\end{aligned}
$$

Hence $M$ satisfies $\left(\mathrm{M}_{2}\right)$ for $p>1, b(p-1)<a, 0<\sigma \leq 1-\frac{b(p-1)}{a}$.

Moreover, $M$ satisfies $\left(\mathrm{M}_{3}\right)$ for $p=2, s=1,0<\sigma \leq 1$ and $\sigma+\mu \leq a-b$.

The rest of the paper is organized as follows. In Section 2, we present some properties of $(\mathrm{PS})_{c}$ sequences. The proofs of Theorems 1.1-1.3 are given in Section 3. Then we establish some properties of the Nehari manifold and give the proofs of Theorems 1.4 and 1.5 in the last section.

\section{Properties of $(\mathrm{PS})_{c}$ sequences}

We say that $\left\{u_{n}\right\}$ is a (PS) ${ }_{c}$ sequence for the functional $J$ if

$$
J\left(u_{n}\right) \rightarrow c \quad \text { and } \quad J^{\prime}\left(u_{n}\right) \rightarrow 0 \quad \text { in } X^{*},
$$

where $X^{*}$ denotes the dual space of $X$. If every $(\mathrm{PS})_{c}$ sequence of $J$ has a strong convergent subsequence, then we say that $J$ satisfies the (PS) condition.

In this section, we derive some results related to the $(\mathrm{PS})_{c}$ sequence.

Lemma 2.1 Assume $\left(\mathrm{f}_{1}\right)$ and $\left(\mathrm{M}_{1}\right)$. Then any bounded $(\mathrm{PS})_{c}$ sequence of $J$ has a strong convergent subsequence. 
Proof The proof is almost the same as Lemma 2.1 in [10], though it was supposed $\left(\tilde{f}_{1}\right)$ $|f(x, t)| \leq C|t|^{q-1}$ instead of $\left(f_{1}\right)$ there.

By Lemma 2.1, in order to get a strong convergent subsequence from any (PS) ${ }_{c}$ sequence of $J$, it suffices to verify the boundedness of the (PS) ${ }_{c}$ sequence. In the following, we present three lemmas about the boundedness of the $(\mathrm{PS})_{c}$ sequence of $J$ under different assumptions on the functions $M$ and $f$.

Lemma 2.2 Assume that $M$ satisfies $\left(\mathrm{M}_{1}\right)-\left(\mathrm{M}_{2}\right)$ and $f$ satisfies $\left(\mathrm{f}_{4}\right)$. Then any $(\mathrm{PS})_{c}$ sequence of the functional $J$ is bounded in $X$.

Proof Let $\left\{u_{n}\right\}$ be a $(\mathrm{PS})_{c}$ sequence of the functional $J$. Then by $\left(\mathrm{M}_{1}\right)-\left(\mathrm{M}_{2}\right)$ and $\left(\mathrm{f}_{4}\right)$, one has

$$
\begin{aligned}
c+1+\left\|u_{n}\right\| \geq & J\left(u_{n}\right)-\frac{1}{\alpha}\left\langle J^{\prime}\left(u_{n}\right), u_{n}\right\rangle \\
= & \frac{1}{p} \hat{M}\left(\left\|u_{n}\right\|^{p}\right)-\int_{\Omega} F\left(x, u_{n}\right) d x-\frac{1}{\alpha}\left[M\left(\left\|u_{n}\right\|^{p}\right)\right]^{p-1}\left\|u_{n}\right\|^{p} \\
& +\frac{1}{\alpha} \int_{\Omega} f\left(x, u_{n}\right) u_{n} d x \\
\geq & \left(\frac{\sigma}{p}-\frac{1}{\alpha}\right)\left[M\left(\left\|u_{n}\right\|^{p}\right)\right]^{p-1}\left\|u_{n}\right\|^{p}-\int_{\Omega}\left(F\left(x, u_{n}\right)-\frac{1}{\alpha} f\left(x, u_{n}\right) u_{n}\right) d x \\
\geq & \left(\frac{\sigma}{p}-\frac{1}{\alpha}\right) m_{0}^{p-1}\left\|u_{n}\right\|^{p} .
\end{aligned}
$$

Therefore, $\left\{u_{n}\right\}$ is bounded in $X$.

Lemma 2.3 If assumptions $\left(\mathrm{M}_{1}\right),\left(\mathrm{M}_{2}\right),\left(\mathrm{f}_{1}\right),\left(\mathrm{f}_{2}\right)$ and $\left(\mathrm{f}_{5}\right)$ are satisfied, then any $(\mathrm{PS})_{c}$ sequence of the functional $J$ is bounded in $X$.

Proof Set $h(t)=F\left(x, t^{-1} z\right) t^{\alpha}, t \in[1, \infty)$. For $|z| \geq r$ and $1 \leq t \leq r^{-1}|z|$, we deduce from $\left(f_{5}\right)$ that

$$
\begin{aligned}
h^{\prime}(t) & =f\left(x, t^{-1} z\right)\left(-z t^{-2}\right) t^{\alpha}+F\left(x, t^{-1} z\right) \alpha t^{\alpha-1} \\
& =t^{\alpha-1}\left[\alpha F\left(x, t^{-1} z\right)-t^{-1} z f\left(x, t^{-1} z\right)\right] \leq 0 .
\end{aligned}
$$

Hence $h(1) \geq h\left(r^{-1}|z|\right)$. Therefore,

$$
F(x, z) \geq r^{-\alpha} F\left(x, r|z|^{-1} z\right)|z|^{\alpha} \geq C_{1}|z|^{\alpha},
$$

where $C_{1}=r^{-\alpha} \inf _{x \in \Omega,|u|=r} F(x, u)>0$. Then there exists $\beta$ such that $\max \left\{\frac{p}{\sigma}, p\right\}<\beta<\alpha$ and

$$
\lim _{|u| \rightarrow \infty} \frac{F(x, u)}{|u|^{\beta}}=+\infty
$$

Let $\left\{u_{n}\right\}$ be a (PS) $)_{c}$ sequence of the functional $J$. In the following, we prove that $\left\{u_{n}\right\}$ is bounded in $X$. Suppose, on the contrary, that $\left\{u_{n}\right\}$ is unbounded. Then we can assume, without loss of generality, that $\left\|u_{n}\right\| \rightarrow \infty$ as $n \rightarrow \infty$. 
By integrating $\left(\mathrm{M}_{2}\right)$, we obtain

$$
\hat{M}(t) \leq \hat{M}\left(t_{0}\right)\left(\frac{t}{t_{0}}\right)^{1 / \sigma}
$$

and so

$$
M(t) \leq\left(\frac{\hat{M}\left(t_{0}\right)}{\sigma t_{0}^{1 / \sigma}}\right)^{\frac{1}{p-1}} t^{\frac{1-\sigma}{\sigma(p-1)}}
$$

holds for all $t \geq t_{0}>0$. Consequently,

$$
\begin{aligned}
\frac{\left[M\left(\left\|u_{n}\right\|^{p}\right)\right]^{p-1}\left\|u_{n}\right\|^{p}}{\left\|u_{n}\right\|^{\beta}} & \leq \frac{\frac{\hat{M}\left(t_{0}\right)}{\sigma t_{0}^{1 / \sigma}}\left\|u_{n}\right\|^{p \frac{1-\sigma}{\sigma}}\left\|u_{n}\right\|^{p}}{\left\|u_{n}\right\|^{\beta}} \\
& =\frac{\hat{M}\left(t_{0}\right)}{\sigma t_{0}^{1 / \sigma}}\left\|u_{n}\right\|^{\frac{p}{\sigma}-\beta} \rightarrow 0 \quad \text { as } n \rightarrow \infty .
\end{aligned}
$$

Note that

$$
\frac{\left\langle J^{\prime}\left(u_{n}\right), u_{n}\right\rangle}{\left\|u_{n}\right\|^{\beta}}=\frac{\left[M\left(\left\|u_{n}\right\|^{p}\right)\right]^{p-1}\left\|u_{n}\right\|^{p}}{\left\|u_{n}\right\|^{\beta}}-\int_{\Omega} \frac{f\left(x, u_{n}\right) u_{n}}{\left\|u_{n}\right\|^{\beta}} d x
$$

we deduce that

$$
\lim _{n \rightarrow \infty} \int_{\Omega} \frac{f\left(x, u_{n}\right) u_{n}}{\left\|u_{n}\right\|^{\beta}} d x=0
$$

Set $v_{n}=\frac{u_{n}}{\left\|u_{n}\right\|}$. Since $X$ is a Banach space and $\left\|v_{n}\right\|=1$, passing to a subsequence if necessary, there is a point $v \in X$ such that

$$
v_{n} \rightarrow v \quad \text { weakly in } X, \quad v_{n} \rightarrow v \quad \text { strongly in } L^{\beta}(\Omega), \quad \text { and } \quad v_{n} \rightarrow v \quad \text { a.e. in } \Omega \text {. }
$$

Denote $\Omega_{0}:=\{x \in \Omega \mid v(x) \neq 0\}$. Then $\left|u_{n}(x)\right| \rightarrow \infty$ for a.e. $x \in \Omega_{0}$. By assumptions $\left(\mathrm{f}_{1}\right),\left(\mathrm{f}_{2}\right)$ and $\left(\mathrm{f}_{5}\right)$, we know that there exist constants $C_{2}, C_{3}>0$ such that

$$
f(x, u) u \geq C_{2}|u|^{\beta}-C_{3}|u|^{p} \quad \text { for all }(x, u) \in \Omega \times \mathbb{R} .
$$

Therefore

$$
\int_{\Omega} \frac{f\left(x, u_{n}\right) u_{n}}{\left\|u_{n}\right\|^{\beta}} d x \geq C_{2} \int_{\Omega}\left|v_{n}\right|^{\beta} d x-C_{3} \int_{\Omega} \frac{\left|v_{n}\right|^{p}}{\left\|u_{n}\right\|^{\beta-p}} d x .
$$

Consequently,

$$
\lim _{n \rightarrow \infty} \int_{\Omega} \frac{f\left(x, u_{n}\right) u_{n}}{\left\|u_{n}\right\|^{\beta}} d x \geq C_{2} \int_{\Omega}|v|^{\beta} d x=C_{2} \int_{\Omega_{0}}|v|^{\beta} d x
$$

If meas $\left(\Omega_{0}\right)>0$, then

$$
0=\lim _{n \rightarrow \infty} \int_{\Omega} \frac{f\left(x, u_{n}\right) u_{n}}{\left\|u_{n}\right\|^{\beta}} d x \geq C_{2} \int_{\Omega_{0}}|v|^{\beta} d x>0
$$


This is a contradiction. Hence meas $\left(\Omega_{0}\right)=0$. So, $v(x)=0$ a.e. in $\Omega$. Moreover, by $\left(f_{1}\right),\left(f_{2}\right)$ and $\left(\mathrm{f}_{5}\right)$ we know that there is a constant $C_{4}>0$ such that

$$
\frac{1}{\alpha} u f(x, u)-F(x, u) \geq-C_{4}|u|^{p} \quad \text { for all }(x, u) \in \Omega \times \mathbb{R} .
$$

Consequently,

$$
\begin{aligned}
\frac{1}{\left\|u_{n}\right\|^{p}} & {\left[J\left(u_{n}\right)-\frac{1}{\alpha}\left\langle J^{\prime}\left(u_{n}\right), u_{n}\right\rangle\right] } \\
\geq & \left(\frac{\sigma}{p}-\frac{1}{\alpha}\right)\left[M\left(\left\|u_{n}\right\|^{p}\right)\right]^{p-1} \\
& -\int_{\Omega}\left(F\left(x, u_{n}\right)-\frac{1}{\alpha} f\left(x, u_{n}\right) u_{n}\right) \frac{1}{\left\|u_{n}\right\|^{p}} d x \\
\geq & \left(\frac{\sigma}{p}-\frac{1}{\alpha}\right) m_{0}^{p-1}-C_{4} \int_{\Omega}\left|v_{n}\right|^{p} d x .
\end{aligned}
$$

This implies $0 \geq\left(\frac{\sigma}{p}-\frac{1}{\alpha}\right) m_{0}^{p-1}$. But this is again impossible. Therefore $\left\{u_{n}\right\}$ is bounded in $X$.

Note that $\alpha>\frac{p}{\sigma}$ in assumptions $\left(\mathrm{f}_{4}\right)$ and $\left(\mathrm{f}_{5}\right)$. Now, we consider the case $\alpha=\frac{p}{\sigma}$. In this case, we should strengthen our assumption on $M$. Then, we have the following result.

Lemma 2.4 Assume that conditions $\left(\mathrm{M}_{1}\right),\left(\mathrm{M}_{3}\right)$ and $\left(f_{6}\right)$ are satisfied. Then any $(\mathrm{PS})_{c}$ sequence of the functional $\mathrm{J}$ is bounded.

Proof It follows from the assumptions that

$$
\begin{aligned}
c+1+\left\|u_{n}\right\| & \geq J\left(u_{n}\right)-\frac{\sigma}{p}\left\langle J^{\prime}\left(u_{n}\right), u_{n}\right\rangle \\
& \geq \frac{\mu}{p}\left\|u_{n}\right\|^{p s}-\int_{\Omega}\left(F\left(x, u_{n}\right)-\frac{\sigma}{p} f\left(x, u_{n}\right) u_{n}\right) d x \\
& \geq \frac{\mu}{p}\left\|u_{n}\right\|^{p s} .
\end{aligned}
$$

Since $p s>1,\left\|u_{n}\right\|$ is bounded in $X$.

\section{Proofs of Theorems 1.1-1.3}

In this section, we use the following fountain theorem to prove Theorems 1.1-1.3.

Lemma 3.1 (Fountain theorem [15]) Let $X$ be a Banach space with the norm $\|\cdot\|$, and let $X_{i}$ be a sequence of subspace of $X$ with $\operatorname{dim} X_{i}<\infty$ for each $i \in \mathbb{N}$. Further, set

$$
X=\overline{\bigoplus_{i=1}^{\infty} X_{i}}, \quad Y_{k}=\bigoplus_{i=1}^{k} X_{i}, \quad Z_{k}=\overline{\bigoplus_{i=k}^{\infty} X_{i}} .
$$

Consider an even functional $\Phi \in C^{1}(X, \mathbb{R})$. Assume that for each $k \in \mathbb{N}$, there exist $\rho_{k}>\gamma_{k}>$ 0 such that 
$\left(\Phi_{1}\right) \quad a_{k}:=\max _{u \in Y_{k},\|u\|=\rho_{k}} \Phi(u) \leq 0$,

$\left(\Phi_{2}\right) \quad b_{k}:=\inf _{u \in Z_{k},\|u\|=\gamma_{k}} \Phi(u) \rightarrow+\infty, k \rightarrow+\infty$,

$\left(\Phi_{3}\right) \Phi$ satisfies the $(\mathrm{PS})_{c}$ condition for every $c>0$.

Then $\Phi$ has an unbounded sequence of critical values.

Proof of Theorem 1.1 Since $X=W_{0}^{1, p}(\Omega)$ is a reflexive and separable Banach space, it is well known that there exist $e_{j} \in X$ and $e_{j}^{*} \in X^{*}(j=1,2, \ldots)$ such that

(1) $\left\langle e_{i}, e_{j}^{*}\right\rangle=\delta_{i j}$, where $\delta_{i j}=1$ for $i=j$ and $\delta_{i j}=0$ for $i \neq j$.

(2) $X=\overline{\operatorname{span}\left\{e_{1}, e_{2}, \ldots\right\}}, X^{*}=\overline{\operatorname{span}\left\{e_{1}^{*}, e_{2}^{*}, \ldots\right\}}$.

Set $X_{i}=\operatorname{span}\left\{e_{i}\right\}, Y_{k}=\bigoplus_{i=1}^{k} X_{i}, Z_{k}=\overline{\bigoplus_{i=k}^{\infty} X_{i}}$.

In the following, we verify that $J$ satisfies all the conditions of the fountain theorem.

1. By $\left(f_{3}\right)$, the energy functional $J$ is even.

2. In view of $\left(\mathrm{f}_{2}\right)$ and $\left(\mathrm{f}_{4}\right)$, there exist positive constants $C_{5}$ and $C_{6}$ such that

$$
F(x, u) \geq C_{5}|u|^{\alpha}-C_{6} \quad \text { for all }(x, u) \in \Omega \times \mathbb{R} .
$$

Moreover, inequality (2.1) implies that there exist constants $C_{7}, C_{8}>0$ such that

$$
\hat{M}(t) \leq C_{7} t^{1 / \sigma}+C_{8}
$$

for all $t \geq 0$. Hence

$$
J(u) \leq \frac{1}{p}\left(C_{7}\|u\|^{\frac{p}{\sigma}}+C_{8}\right)-\int_{\Omega}\left(C_{5}|u|^{\alpha}-C_{6}\right) d x .
$$

Since all norms are equivalent on the finite dimensional space $Y_{k}$ and $\alpha>\frac{p}{\sigma}$, we have

$$
a_{k}:=\max _{u \in Y_{k},\|u\|=\rho_{k}} J(u)<0
$$

for $\|u\|=\rho_{k}$ sufficiently large.

3. Set $\beta_{k}=\sup _{u \in Z_{k},\|u\|=1}\left(\int_{\Omega}|u|^{q} d x\right)^{1 / q}$. From the fact $Z_{k+1} \subset Z_{k}$, it is clear that $0 \leq \beta_{k+1} \leq$ $\beta_{k}$. Hence $\beta_{k} \rightarrow \beta_{0} \geq 0$ as $k \rightarrow+\infty$. By the definition of $\beta_{k}$, there exists $u_{k} \in Z_{k}$ with $\left\|u_{k}\right\|=1$ such that

$$
-1 / k \leq \beta_{k}-\left(\int_{\Omega}\left|u_{k}\right|^{q} d x\right)^{1 / q} \leq 0
$$

for all $k \geq 1$. Then there exists a subsequence of $\left\{u_{k}\right\}$ (not relabeled) such that $u_{k} \rightarrow u$ in $X$ and $\left\langle u, e_{j}^{*}\right\rangle=\lim _{k \rightarrow \infty}\left\langle u_{k}, e_{j}^{*}\right\rangle=0$ for all $j \geq 1$. Thus $u=0$. This shows $u_{k} \rightarrow 0$ in $X$ and so $u_{k} \rightarrow 0$ in $L^{q}(\Omega)$. Thus $\beta_{0}=0$.

For any $\epsilon>0,\left(f_{1}\right)$ and $\left(f_{2}\right)$ imply

$$
|F(x, u)| \leq \epsilon|u|^{p}+C(\epsilon)|u|^{q}
$$


for some $C(\epsilon)>0$. Therefore, for any $u \in Z_{k}$, there holds

$$
\begin{aligned}
J(u) & \geq \frac{1}{p} \sigma\left[M\left(\|u\|^{p}\right)\right]^{p-1}\|u\|^{p}-\int_{\Omega} F(x, u) d x \\
& \geq \frac{\sigma}{p} m_{0}^{p-1}\|u\|^{p}-\epsilon \int_{\Omega}|u|^{p} d x-C(\epsilon) \int_{\Omega}|u|^{q} d x \\
& \geq\left(\frac{\sigma}{p} m_{0}^{p-1}-\epsilon S_{p}^{-1}\right)\|u\|^{p}-C(\epsilon) \beta_{k}^{q}\|u\|^{q}
\end{aligned}
$$

where $S_{p}$ is the best Sobolev constant for the embedding of $X$ into $L^{p}(\Omega)$, i.e.,

$$
\|u\|_{L^{p}(\Omega)} \leq S_{p}^{-1 / p}\|u\|
$$

Select $\epsilon$ so small that $\frac{\sigma}{p} m_{0}^{p-1}-\epsilon S_{p}^{-1}>0$ and let

$$
\gamma_{k}=\left(\frac{\frac{\sigma}{p} m_{0}^{p-1}-\epsilon S_{p}^{-1}}{2 C(\epsilon) \beta_{k}^{q}}\right)^{\frac{1}{q-p}}
$$

we obtain

$$
b_{k}:=\inf _{u \in Z_{k},\|u\|=\gamma_{k}} J(u) \geq \frac{1}{2}\left(\frac{\sigma}{p} m_{0}^{p-1}-\epsilon S_{p}^{-1}\right) \gamma_{k}^{p} .
$$

Since $\beta_{k} \rightarrow 0$, we have $b_{k} \rightarrow+\infty$ as $k \rightarrow+\infty$.

4. By Lemmas 2.1 and 2.2, $J$ satisfies the $(\mathrm{PS})_{c}$ condition. Consequently, the conclusion follows from the fountain theorem.

Proof of Theorem 1.2 It follows from Lemmas 2.1 and 2.3 that $J$ satisfies the (PS) ${ }_{c}$ condition. Similar to the proof of Theorem 1.1, we have that all the conditions of Lemma 3.1 are fulfilled.

Proof of Theorem 1.3 By Lemmas 2.1 and 2.4 , $J$ satisfies the $(\mathrm{PS})_{c}$ condition. From the proof of Theorem 1.1, it is sufficient to show that condition $\left(\Phi_{1}\right)$ in Lemma 3.1 is satisfied.

By $\left(f_{1}\right),\left(f_{2}\right)$ and $\left(f_{7}\right)$, we deduce that for any $M>0$, there exists a constant $C(M)>0$ such that

$$
F(x, u) \geq M|u|^{\frac{p}{\sigma}}-C(M)
$$

Since $\left(M_{3}\right)$ implies $\left(M_{2}\right)$, it follows that (3.1) still holds. Therefore

$$
J(u) \leq \frac{1}{p}\left(C_{7}\|u\|^{\frac{p}{\sigma}}+C_{8}\right)-\int_{\Omega}\left(M|u|^{\frac{p}{\sigma}}-C(M)\right) d x .
$$

Note that all norms are equivalent on the finite dimensional space $Y_{k}$, there exists a constant $\mu_{1}>0$ such that

$$
\begin{aligned}
J(u) & \leq \frac{1}{p}\left(C_{7}\|u\|^{\frac{p}{\sigma}}+C_{8}\right)-\mu_{1} M\|u\|^{\frac{p}{\sigma}}+C(M)|\Omega| \\
& =\left(\frac{C_{7}}{p}-\mu_{1} M\right)\|u\|^{\frac{p}{\sigma}}+\frac{C_{8}}{p}+C(M)|\Omega| .
\end{aligned}
$$


Fix $M>\frac{C_{7}}{p \mu_{1}}$, then there exists large $\rho_{k}>0$ such that

$$
a_{k}:=\max _{u \in Y_{k},\|u\|=\rho_{k}} J(u)<0
$$

This completes the proof.

\section{Proofs of Theorems 1.4 and 1.5}

In this section, we consider a special case $f(x, u)=\lambda|u|^{q-2} u+|u|^{r-2} u\left(1<q<p<r<p^{*}\right)$. In this case, the associated energy functional is

$$
J_{\lambda}(u)=\frac{1}{p} \hat{M}\left(\|u\|^{p}\right)-\frac{1}{q} \int_{\Omega} \lambda|u|^{q} d x-\frac{1}{r} \int_{\Omega}|u|^{r} d x
$$

where $\hat{M}(s)=\int_{0}^{s}[M(t)]^{p-1} d t$. It is well known that the energy functional $J_{\lambda}(u)$ is of class $C^{1}$ in $X=H_{0}^{1}(\Omega)$ and the solutions of problem (1.1) are the critical points of the energy functional. Since $J_{\lambda}$ is not bounded below on $X$, it is useful to consider the problem on the Nehari manifold

$$
\mathcal{N}=\left\{u \in X \backslash\{0\} \mid\left\langle J_{\lambda}^{\prime}(u), u\right\rangle=0\right\}
$$

where $\langle\cdot, \cdot\rangle$ denotes the usual duality. Clearly, $u \in \mathcal{N}$ if and only if

$$
\left[M\left(\|u\|^{p}\right)\right]^{p-1}\|u\|^{p}=\int_{\Omega} \lambda|u|^{q} d x+\int_{\Omega}|u|^{r} d x .
$$

Since $\mathcal{N}$ is a much smaller set than $X$, it is easier to study $J_{\lambda}(u)$ on the Nehari manifold. Moreover, we have the following result.

Lemma 4.1 Assume $\sigma r>p$ and $M$ satisfies $\left(\mathrm{M}_{1}\right),\left(\mathrm{M}_{2}\right)$. Then the energy functional $J_{\lambda}$ is coercive and bounded below on $\mathcal{N}$.

Proof We denote by $C_{s}$ the best Sobolev constant for the embedding of $X$ in $L^{s}(\Omega)$ with $1<s<p^{*}$. In particular,

$$
\|u\|_{L^{s}(\Omega)} \leq C_{s}^{-1 / p}\|u\| \quad \text { for all } u \in X \backslash\{0\}
$$

Let $u \in \mathcal{N}$. Then we have

$$
\begin{aligned}
J_{\lambda}(u) & =\frac{1}{p} \hat{M}\left(\|u\|^{p}\right)-\frac{1}{q} \int_{\Omega} \lambda|u|^{q} d x-\frac{1}{r} \int_{\Omega}|u|^{r} d x \\
& \geq \frac{1}{p} \sigma\left[M\left(\|u\|^{p}\right)\right]^{p-1}\|u\|^{p}-\frac{1}{q} \int_{\Omega} \lambda|u|^{q} d x-\frac{1}{r}\left\{\left[M\left(\|u\|^{p}\right)\right]^{p-1}\|u\|^{p}-\int_{\Omega} \lambda|u|^{q} d x\right\} \\
& =\left(\frac{\sigma}{p}-\frac{1}{r}\right)\left[M\left(\|u\|^{p}\right)\right]^{p-1}\|u\|^{p}-\lambda\left(\frac{1}{q}-\frac{1}{r}\right) \int_{\Omega}|u|^{q} d x \\
& \geq\left(\frac{\sigma}{p}-\frac{1}{r}\right) m_{0}^{p-1}\|u\|^{p}-\lambda\left(\frac{1}{q}-\frac{1}{r}\right) C_{q}^{-\frac{q}{p}}\|u\|^{q} .
\end{aligned}
$$

Since $\frac{\sigma}{p}>\frac{1}{r}$ and $q<p<r, J_{\lambda}$ is coercive and bounded below on $\mathcal{N}$. 
The Nehari manifold $\mathcal{N}$ is closely linked to the behavior of the fibering map $K_{u}: t \rightarrow$ $J_{\lambda}(t u)$. For $u \in X$, we have

$$
\begin{aligned}
K_{u}(t)= & \frac{1}{p} \hat{M}\left(t^{p}\|u\|^{p}\right)-\frac{1}{q} t^{q} \int_{\Omega} \lambda|u|^{q} d x-\frac{1}{r} t^{r} \int_{\Omega}|u|^{r} d x \\
K_{u}^{\prime}(t)= & {\left[M\left(t^{p}\|u\|^{p}\right)\right]^{p-1} t^{p-1}\|u\|^{p}-\lambda t^{q-1} \int_{\Omega}|u|^{q} d x-t^{r-1} \int_{\Omega}|u|^{r} d x } \\
K_{u}^{\prime \prime}(t)= & {\left[M\left(t^{p}\|u\|^{p}\right)\right]^{p-1}(p-1) t^{p-2}\|u\|^{p} } \\
& +p(p-1) t^{2 p-2}\|u\|^{2 p}\left[M\left(t^{p}\|u\|^{p}\right)\right]^{p-2} M^{\prime}\left(t^{p}\|u\|^{p}\right) \\
& -\lambda(q-1) t^{q-2} \int_{\Omega}|u|^{q} d x-(r-1) t^{r-2} \int_{\Omega}|u|^{r} d x
\end{aligned}
$$

Clearly, $t u \in \mathcal{N}$ if and only if $K_{u}^{\prime}(t)=0$. It is natural to split $\mathcal{N}$ into three parts corresponding to local minima, local maxima and points of inflection, i.e.,

$$
\begin{aligned}
& \mathcal{N}^{+}=\left\{u \in \mathcal{N} \mid K_{u}^{\prime \prime}(1)>0\right\}, \\
& \mathcal{N}^{0}=\left\{u \in \mathcal{N} \mid K_{u}^{\prime \prime}(1)=0\right\}, \\
& \mathcal{N}^{-}=\left\{u \in \mathcal{N} \mid K_{u}^{\prime \prime}(1)<0\right\} .
\end{aligned}
$$

Then we have the following lemmas.

Lemma 4.2 Suppose that $u_{0}$ is a local minimizer of $J_{\lambda}$ on $\mathcal{N}$ and $u_{0} \notin \mathcal{N}^{0}$. Then $u_{0}$ is a critical point of $J_{\lambda}$.

Proof Our proof is almost the same as that of Binding et al. [16] and Brown and Zhang [17].

Lemma 4.3 Suppose that $M$ satisfies $\left(\mathrm{M}_{1}\right)$ and $\left(\mathrm{M}_{4}\right)$. Then there exists $\lambda_{0}>0$ such that $\mathcal{N}^{0}=\emptyset$ for all $0<\lambda<\lambda_{0}$.

Proof For each $u \in \mathcal{N}$, we have

$$
\begin{aligned}
K_{u}^{\prime \prime}(1)= & (p-q)\left[M\left(\|u\|^{p}\right)\right]^{p-1}\|u\|^{p}+p(p-1)\|u\|^{2 p}\left[M\left(\|u\|^{p}\right)\right]^{p-2} M^{\prime}\left(\|u\|^{p}\right) \\
& -(r-q) \int_{\Omega}|u|^{r} d x \\
= & -(r-p)\left[M\left(\|u\|^{p}\right)\right]^{p-1}\|u\|^{p}+p(p-1)\|u\|^{2 p}\left[M\left(\|u\|^{p}\right)\right]^{p-2} M^{\prime}\left(\|u\|^{p}\right) \\
& +\lambda(r-q) \int_{\Omega}|u|^{q} d x .
\end{aligned}
$$

Furthermore, if $u \in \mathcal{N}^{0}$, then

$$
\begin{aligned}
(p-q) m_{0}^{p-1}\|u\|^{p} & \leq(p-q)\left[M\left(\|u\|^{p}\right)\right]^{p-1}\|u\|^{p}+p(p-1)\|u\|^{2 p}\left[M\left(\|u\|^{p}\right)\right]^{p-2} M^{\prime}\left(\|u\|^{p}\right) \\
& =(r-q) \int_{\Omega}|u|^{r} d x \leq(r-q) C_{r}^{-\frac{r}{p}}\|u\|^{r}
\end{aligned}
$$


and

$$
\begin{aligned}
\frac{(r-p)(d-1)}{d} m_{0}^{p-1}\|u\|^{p} \leq & \frac{(r-p)(d-1)}{d}\left[M\left(\|u\|^{p}\right)\right]^{p-1}\|u\|^{p} \\
\leq & (r-p)\left[M\left(\|u\|^{p}\right)\right]^{p-1}\|u\|^{p} \\
& -p(p-1)\|u\|^{2 p}\left[M\left(\|u\|^{p}\right)\right]^{p-2} M^{\prime}\left(\|u\|^{p}\right) \\
\leq & \lambda(r-q) C_{q}^{-\frac{q}{p}}\|u\|^{q} .
\end{aligned}
$$

Consequently,

$$
\left(\frac{(p-q) m_{0}^{p-1}}{(r-q) C_{r}^{-r / p}}\right)^{1 /(r-p)} \leq\|u\| \leq\left(\frac{\lambda d(r-q) C_{q}^{-q / p}}{(r-p)(d-1) m_{0}^{p-1}}\right)^{1 /(p-q)} .
$$

Therefore,

$$
\lambda \geq \lambda_{0}:=\left(\frac{(p-q) m_{0}^{p-1}}{(r-q) C_{r}^{-r / p}}\right)^{(p-q) /(r-p)} \frac{(r-p)(d-1) m_{0}^{p-1}}{d(r-q) C_{q}^{-q / p}} .
$$

Hence $\mathcal{N}^{0}=\emptyset$ for all $0<\lambda<\lambda_{0}$.

Lemma 4.4 Suppose that conditions $\left(\mathrm{M}_{1}\right),\left(\mathrm{M}_{2}\right)$ hold. Assume also $0<\lambda<\lambda_{0} \frac{d}{d-1}$ and $q<$ $\frac{p}{\sigma}<r$. Then, for each $u \in X \backslash\{0\}$, there exist $t^{+}$and $t^{-}$such that $t^{+} u \in \mathcal{N}^{+}$and $t^{-} u \in \mathcal{N}^{-}$.

Proof Fix $u \in X \backslash\{0\}$. Then it follows from condition $\left(\mathrm{M}_{1}\right)$ that

$$
\begin{aligned}
K_{u}^{\prime}(t) & =\left[M\left(t^{p}\|u\|^{p}\right)\right]^{p-1} t^{p-1}\|u\|^{p}-\lambda t^{q-1} \int_{\Omega}|u|^{q} d x-t^{r-1} \int_{\Omega}|u|^{r} d x \\
& \geq m_{0}^{p-1} t^{p-1}\|u\|^{p}-\lambda t^{q-1} \int_{\Omega}|u|^{q} d x-t^{r-1} \int_{\Omega}|u|^{r} d x \\
& =t^{p-1}\left(m_{0}^{p-1}\|u\|^{p}-h(t)\right),
\end{aligned}
$$

where $h(t)=\lambda t^{q-p} \int_{\Omega}|u|^{q} d x+t^{r-p} \int_{\Omega}|u|^{r} d x$. Since

$$
h^{\prime}(t)=\lambda(q-p) t^{q-p-1} \int_{\Omega}|u|^{q} d x+(r-p) t^{r-p-1} \int_{\Omega}|u|^{r} d x
$$

we obtain $h^{\prime}\left(t_{M}\right)=0$ for

$$
t_{M}=\left(\frac{\lambda(p-q) \int_{\Omega}|u|^{q} d x}{(r-p) \int_{\Omega}|u|^{r} d x}\right)^{\frac{1}{r-q}}
$$

Moreover,

$$
\begin{aligned}
h\left(t_{M}\right) & =\left(\frac{r-p}{p-q}+1\right) t_{M}^{r-p} \int_{\Omega}|u|^{r} d x \\
& =\frac{r-q}{p-q}\left(\frac{\lambda(p-q) \int_{\Omega}|u|^{q} d x}{(r-p) \int_{\Omega}|u|^{r} d x}\right)^{\frac{r-p}{r-q}} \int_{\Omega}|u|^{r} d x
\end{aligned}
$$




$$
\begin{aligned}
& =\frac{r-q}{p-q}\left(\frac{\lambda(p-q)}{r-p}\right)^{\frac{r-p}{r-q}}\left(\int_{\Omega}|u|^{q} d x\right)^{\frac{r-p}{r-q}}\left(\int_{\Omega}|u|^{r} d x\right)^{\frac{p-q}{r-q}} \\
& \leq \frac{r-q}{p-q}\left(\frac{\lambda(p-q)}{r-p}\right)^{\frac{r-p}{r-q}} C_{q}^{-\frac{q(r-p)}{p(r-q)}} C_{r}^{-\frac{r(p-q)}{p(r-q)}}\|u\|^{p} .
\end{aligned}
$$

Hence $m_{0}^{p-1}\|u\|^{p}>h\left(t_{M}\right)$ and so $K_{u}^{\prime}\left(t_{M}\right)>0$ for all

$$
0<\lambda<m_{0}^{(p-1) \frac{r-q}{r-p}} C_{q}^{q / p} C_{r}^{\frac{r(p-q)}{p(r-p)}} \frac{r-p}{p-q}\left(\frac{p-q}{r-q}\right)^{\frac{r-q}{r-p}}=\lambda_{0} \frac{d}{d-1} .
$$

On the other hand, it follows from (2.2) that

$$
\begin{aligned}
K_{u}^{\prime}(t) & =\left[M\left(t^{p}\|u\|^{p}\right)\right]^{p-1} t^{p-1}\|u\|^{p}-\lambda t^{q-1} \int_{\Omega}|u|^{q} d x-t^{r-1} \int_{\Omega}|u|^{r} d x \\
& \leq \frac{\hat{M}\left(t_{0}\right)}{\sigma t_{0}^{1 / \sigma}}\|u\|^{\frac{p}{\sigma}} t^{\frac{p}{\sigma}-1}-\lambda t^{q-1} \int_{\Omega}|u|^{q} d x-t^{r-1} \int_{\Omega}|u|^{r} d x .
\end{aligned}
$$

Since $q<\frac{p}{\sigma}<r$, there exist $0<t_{1}<t_{M}<t_{2}$ such that $K_{u}^{\prime}\left(t_{1}\right)<0, K_{u}^{\prime}\left(t_{2}\right)<0$. Note that $\mathcal{N}^{0}=\emptyset$, we deduce that there exist $t^{+}, t^{-}$such that $K_{u}^{\prime}\left(t^{+}\right)=K_{u}^{\prime}\left(t^{-}\right)=0$ and $K_{u}^{\prime \prime}\left(t^{+}\right)>0>K_{u}^{\prime \prime}\left(t^{-}\right)$. Hence $t^{+} u \in \mathcal{N}^{+}$and $t^{-} u \in \mathcal{N}^{-}$.

Proof of Theorem 1.4 By Lemma 4.3, we write $\mathcal{N}=\mathcal{N}^{+} \cup \mathcal{N}^{-}$and define

$$
\alpha_{\lambda}^{+}=\inf _{u \in \mathcal{N}^{+}} J_{\lambda}(u), \quad \alpha_{\lambda}^{-}=\inf _{u \in \mathcal{N}^{-}} J_{\lambda}(u)
$$

In view of Lemma 4.1 and the Ekeland variational principle [18], there exist minimizing sequences $\left\{u_{n}^{+}\right\}$and $\left\{u_{n}^{-}\right\}$for $J_{\lambda}$ on $\mathcal{N}^{+}$and $\mathcal{N}^{-}$such that

$$
J_{\lambda}\left(u_{n}^{+}\right)=\alpha_{\lambda}^{+}+o(1), \quad J_{\lambda}\left(u_{n}^{-}\right)=\alpha_{\lambda}^{-}+o(1)
$$

and

$$
J_{\lambda}^{\prime}\left(u_{n}^{+}\right)=o(1), \quad J_{\lambda}^{\prime}\left(u_{n}^{-}\right)=o(1)
$$

Furthermore, Lemma 2.1 implies that there exist $u_{0}^{+}$and $u_{0}^{-}$such that $u_{n}^{+} \rightarrow u_{0}^{+}$and $u_{n}^{-} \rightarrow u_{0}^{-}$ strongly in $X$. Note that $u_{n}^{+} \in \mathcal{N}^{+}$implies $K_{u_{n}^{+}}^{\prime}(1)=0$ and $K_{u_{n}^{+}}^{\prime \prime}(1)>0$. Letting $n \rightarrow \infty$, we deduce that $K_{u^{+}}^{\prime}(1)=0$ and $K_{u^{+}}^{\prime \prime}(1) \geq 0$, and so $u^{+} \in \mathcal{N}^{+} \cup \mathcal{N}^{0}$. By Lemma 4.3, we obtain $u^{+} \in \mathcal{N}^{+}$. Similarly, $u^{-} \in \mathcal{N}^{-}$. Since $J_{\lambda}(u)=J_{\lambda}(|u|)$, we may assume $u_{0}^{+}$and $u_{0}^{-}$are nonnegative. Moreover, it can be deduced from Lemma 4.2 that $u_{0}^{+}$and $u_{0}^{-}$are nonnegative solutions of equation (1.1). Finally, since $\mathcal{N}^{+} \cap \mathcal{N}^{-}=\emptyset$, we infer that $u_{0}^{+}$and $u_{0}^{-}$are two distinct solutions.

Finally, we prove Theorem 1.5 by the following dual fountain theorem.

Theorem 4.1 (Dual fountain theorem [19]) Assume that $J \in C^{1}(X, \mathbb{R})$ satisfies $J(-u)=J(u)$. If for every $k \in \mathbb{N}$ there exist $\rho_{k}>r_{k}>0$ such that

$\left(\mathrm{B}_{1}\right) \quad a_{k}:=\inf _{u \in Z_{k},\|u\|=\rho_{k}} J(u) \geq 0$ as $k \rightarrow \infty$, 
$\left(\mathrm{B}_{2}\right) \quad b_{k}:=\max _{u \in Y_{k},\|u\|=r_{k}} J(u)<0$,

$\left(\mathrm{B}_{3}\right) d_{k}:=\inf _{u \in Z_{k},\|u\| \leq \rho_{k}} J(u) \rightarrow 0$ as $k \rightarrow \infty$,

$\left(\mathrm{B}_{4}\right) J$ satisfies the $(\mathrm{PS})_{c}^{*}$ condition for every $c \in\left[d_{k_{0}}, 0\right)$, that is, any sequence $\left\{u_{n_{j}}\right\} \subset X$ such that

$$
u_{n_{j}} \in Y_{n_{j}}, \quad J\left(u_{n_{j}}\right) \rightarrow c,\left.\quad J\right|_{Y_{n_{j}}} ^{\prime} \rightarrow 0, \quad \text { as } n_{j} \rightarrow \infty
$$

has a convergent subsequence.

Then $J$ has a sequence of negative critical points $\left\{u_{k}\right\}$ with $J\left(u_{k}\right) \rightarrow 0$.

Proof of Theorem 1.5 1. Let

$$
\beta_{k}:=\sup _{u \in Z_{k},\|u\|=1}\left(\int_{\Omega}|u|^{q} d x\right)^{1 / q} .
$$

Then by $\left(\mathrm{M}_{1}\right)-\left(\mathrm{M}_{2}\right)$, for all $u \in Z_{k}$, there holds

$$
\begin{aligned}
J_{\lambda}(u) & =\frac{1}{p} \hat{M}\left(\|u\|^{p}\right)-\frac{1}{q} \int_{\Omega} \lambda|u|^{q} d x-\frac{1}{r} \int_{\Omega}|u|^{r} d x \\
& \geq \frac{1}{p} \sigma m_{0}^{p-1}\|u\|^{p}-\frac{\lambda}{q} \beta_{k}^{q}\|u\|^{q}-\frac{1}{r} C_{r}^{-\frac{r}{p}}\|u\|^{r} .
\end{aligned}
$$

Since $p<r$, we have

$$
\frac{1}{2 p} \sigma m_{0}^{p-1}\|u\|^{p} \geq \frac{1}{r} C_{r}^{-\frac{r}{p}}\|u\|^{r} \quad \text { for all }\|u\| \leq R=\left(\frac{\sigma r C_{r}^{r / p} m_{0}^{p-1}}{2 p}\right)^{1 /(r-p)} .
$$

Therefore,

$$
J_{\lambda}(u) \geq \frac{1}{2 p} \sigma m_{0}^{p-1}\|u\|^{p}-\frac{\lambda}{q} \beta_{k}^{q}\|u\|^{q} \quad \text { for all } u \in Z_{k} \text { with }\|u\| \leq R .
$$

Choose

$$
\rho_{k}=\left(\frac{2 p \lambda \beta_{k}^{q}}{q \sigma m_{0}^{p-1}}\right)^{1 /(p-q)} .
$$

It follows from $\beta_{k} \rightarrow 0$ that $\rho_{k} \rightarrow 0$. Hence there exists $k_{0}>0$ such that $\rho_{k} \leq R$ for all $k>k_{0}$. Consequently, $J_{\lambda}(u) \geq 0$ for all $k>k_{0}, u \in Z_{k}$ and $\|u\|=\rho_{k}$. This gives $\left(\mathrm{B}_{1}\right)$.

2. Since in the finite dimensional space $Y_{k}$ all norms are equivalent, there exist positive constants $C_{9}, C_{10}$ such that

$$
\int_{\Omega}|u|^{q} d x \geq C_{9}\|u\|^{q} \quad \text { and } \quad \int_{\Omega}|u|^{r} d x \geq C_{10}\|u\|^{r} .
$$

Then, by (2.1), we obtain for all $u \in Y_{k}$

$$
J_{\lambda}(u) \leq \frac{\hat{M}\left(t_{0}\right)}{p t_{0}^{1 / \sigma}}\|u\|^{\frac{p}{\sigma}}-\frac{\lambda}{q} C_{9}\|u\|^{q}-\frac{C_{10}}{r}\|u\|^{r} .
$$


Notice that $\frac{p}{\sigma}>q$ and $r>q$, we deduce that $J_{\lambda}(u)<0$ for $\|u\|=r_{k}$ sufficiently small and $\left(\mathrm{B}_{2}\right)$ is proved.

3. It follows from (4.4) that, for all $u \in Z_{k}$ with $\|u\| \leq \rho_{k}$ and $k>k_{0}$,

$$
J_{\lambda}(u) \geq-\frac{\lambda}{q} \beta_{k}^{q} \rho_{k}^{q}
$$

Since $\beta_{k} \rightarrow 0$ and $\rho_{k} \rightarrow 0$ as $k \rightarrow \infty$, relation $\left(\mathrm{B}_{3}\right)$ is satisfied.

4. Finally, we prove that $J_{\lambda}$ satisfies the (PS)* condition. Let $\left\{u_{n_{j}}\right\}$ be a sequence such that $\left\{u_{n_{j}}\right\} \subset Y_{n_{j}}, J_{\lambda}\left(u_{n_{j}}\right) \rightarrow c$ and $\left.J\right|_{Y_{n_{j}}} ^{\prime} \rightarrow 0$ as $n_{j} \rightarrow \infty$. Then by $\left(\mathrm{M}_{1}\right)-\left(\mathrm{M}_{2}\right)$ we have

$$
\begin{aligned}
c+1+\left\|u_{n_{j}}\right\| & \geq J_{\lambda}\left(u_{n_{j}}\right)-\frac{1}{r}\left\langle J_{\lambda}^{\prime}\left(u_{n_{j}}\right), u_{n_{j}}\right\rangle \\
& =\frac{1}{p} \hat{M}\left(\left\|u_{n_{j}}\right\|^{p}\right)-\frac{1}{r}\left[M\left(\left\|u_{n_{j}}\right\|^{p}\right)\right]^{p-1}\left\|u_{n_{j}}\right\|^{p}-\lambda\left(\frac{1}{q}-\frac{1}{r}\right) \int_{\Omega}\left|u_{n_{j}}\right|^{q} d x \\
& \geq\left(\frac{\sigma}{p}-\frac{1}{r}\right) m_{0}^{p-1}\left\|u_{n_{j}}\right\|^{p}-\lambda\left(\frac{1}{q}-\frac{1}{r}\right) C_{q}^{-q / p}\left\|u_{n_{j}}\right\|^{q} .
\end{aligned}
$$

This implies $\left\|u_{n_{j}}\right\|$ is bounded. Obviously, $f$ satisfies $\left(\mathrm{f}_{1}\right)$. Hence, by Lemma $2.1, J_{\lambda}$ satisfies the $(\mathrm{PS})_{c}^{*}$ condition.

We complete the proof by applying the dual fountain theorem.

\section{Competing interests}

The authors declare that they have no competing interests.

\section{Authors' contributions}

All authors contributed equally to the writing of this paper. All authors read and approved the final manuscript.

\section{Author details}

${ }^{1}$ Math and Physics Teaching Department, Hohai University, Changzhou, 213022, China. ${ }^{2}$ School of Mathematics and Physics, Jiangsu University of Technology, Changzhou, 213015, China.

\section{Acknowledgements}

The authors are grateful for the referee's helpful suggestions and comments. This work is supported by the Fundamental Research Funds for the Central Universities (2016B07514).

\section{Publisher's Note}

Springer Nature remains neutral with regard to jurisdictional claims in published maps and institutional affiliations.

Received: 21 January 2017 Accepted: 20 March 2017 Published online: 24 March 2017

\section{References}

1. Lions, JL: On some questions in boundary value problems of mathematical physics. In: Contemporary Development in Continuum Mechanics and Partial Differential Equations. North-Holland Math. Stud., vol. 30, pp. 284-346. North-Holland, Amsterdam (1978)

2. $\mathrm{Wu}, \mathrm{X}$ : Existence of nontrivial solutions and high energy solutions for Schrodinger-Kirchhoff-type equations in $\mathbb{R}^{N}$. Nonlinear Anal., Real World Appl. 12, 1278-1287 (2011)

3. Nie, J, Wu, X: Existence and multiplicity of non-trivial solutions for Schrodinger-Kirchhoff-type equations with radial potential. Nonlinear Anal. 75, 3470-3479 (2012)

4. Chen, $\mathrm{C}, \mathrm{Kuo}, \mathrm{Y}, \mathrm{Wu}, \mathrm{T}$ : The Nehari manifold for a Kirchhoff type problem involving sign-changing weight functions. J. Differ. Equ. 250, 1876-1908 (2011)

5. Jin, J, Wu, X: Infinitely many radial solutions for Kirchhoff-type problems in $\mathbb{R}^{N}$. J. Math. Anal. Appl. 369, 564-574 (2010)

6. Chen, C, Zhu, Q: Existence of positive solutions to $p$-Kirchhoff-type problem without compactness conditions. Appl. Math. Lett. 28, 82-87 (2014)

7. Huang, J, Chen, C, Xiu, Z: Existence and multiplicity results for a $p$-Kirchhoff equation with a concave-convex term. Appl. Math. Lett. 26, 1070-1075 (2013)

8. Sun, J, Tang, C: Existence and multiplicity of solutions for Kirchhoff type equations. Nonlinear Anal. 74, 1212-1222 (2011) 
9. Alves, CO, Corrêa, FJSA, Ma, TF: Positive solutions for a quasilinear elliptic equation of Kirchhoff type. Comput. Math. Appl. 49, 85-93 (2005)

10. Corrêa, FJSA, Figueiredo, GM: On an elliptic equation of $p$-Kirchhoff type via variational methods. Bull. Aust. Math. Soc. 74(2), 263-277 (2006)

11. Liu, D: On a $p$-Kirchhoff equation via fountain theorem and dual fountain theorem. Nonlinear Anal. 72, 302-308 (2010)

12. Figueiredo, GM, Nascimento, RG: Existence of a nodal solution with minimal energy for a Kirchhoff equation. Math. Nachr. 288(1), 48-60 (2015)

13. Santos, JR Jr.: The effect of the domain topology on the number of positive solutions of an elliptic Kirchhoff problem. Nonlinear Anal., Real World Appl. 28, 269-283 (2016)

14. Hamydy, A, Massar, M, Tsouli, N: Existence of solutions for $p$-Kirchhoff type problems with critical exponent. Electron. J. Differ. Equ. 2011, 105 (2011)

15. Willem, M: Minimax Theorem. Birkhäuser Boston, Boston (1996)

16. Binding, PA, Drabek, P, Huang, YX: On Neumann boundary value problems for some quasilinear elliptic equations Electron. J. Differ. Equ. 1997, 5 (1997)

17. Brown, KJ, Zhang, Y: The Nehari manifold for a semilinear elliptic equation with a sign-changing weight function. J. Differ. Equ. 193, 481-499 (2003)

18. Ekeland, I: On the variational principle. J. Math. Anal. Appl. 47, 324-353 (1974)

19. Bartsch, T, Willem, M: On an elliptic equation with concave and convex nonlinearities. Proc. Am. Math. Soc. 123, 3555-3561 (1995)

\section{Submit your manuscript to a SpringerOpen ${ }^{\ominus}$ journal and benefit from:}

- Convenient online submission

Rigorous peer review

- Immediate publication on acceptance

- Open access: articles freely available online

- High visibility within the field

- Retaining the copyright to your article 